\title{
Multi-walled carbon nanotubes enhanced superhydrophobic M W CNTs-Co/a-C:H carbon-based film for excellent self-cleaning and corrosion resistance
}

\author{
Shengguo Zhou ${ }^{1, *}$, Xiaobo Zhu ${ }^{1}$, Qingqing Yan ${ }^{1}$, Shuncai Wang ${ }^{2}$ \\ ${ }^{1}$ School of M aterial Science and Engineering, J iangxi U niversity of Science and \\ Technology, Ganzhou 341000, PR China \\ 2 national $C$ entre for Advanced Tribology at Southampton (nCATS), School of \\ Engineering Sciences, University of Southampton, S017 1BJ , U K
}

\begin{abstract}
: .
A robust superhydrophobic MW CNTs-Co/a-C:H carbon-based film was successfully fabricated for the first time by a safe technology, namely electrochemical deposition. The multi-walled carbon nanotubes (M W CNTs) and Co nano-particles were confirmed to tailor a micro-nanoscale hierarchical surface and nanocrystallite/amorphous microstructure. The resulting WCNTs-Co/a-C:H carbon-based film exhibited complete water repellency with the contact angle $\left(158.1^{\circ}\right)$ and much smaller sliding angle $\left(2.98^{\circ}\right)$, fairly strong mechanical property, excellent corrosion resistance and self-cleaning ability. This research highlights a promising route for the preparation of robust superhydrophobic film, providing potential uses including self-cleaning, antifouling and anti-corrosion applications such as ship hulls and aeroplane wings.
\end{abstract}

Keywords: Superhydrophobic; M W CNTs-Co/a-C:H; Corrosion resistance; Self-cleaning

\footnotetext{
* Correspondence author. Tel.: +86 13426555692.

E-mail: zhoucreed@163.com/zhousg@jxust.edu.cn
} 


\section{Introduction}

Superhydrophobic surfaces with a minimum $150^{\circ}$. contact angle (CA) and a maximum $10^{\circ}$. sliding angle (SA) for water droplets have attracted extensive attention due to their great significance in both fundamental research and potential applications in the fields of oilwater separation [1-4], water collection [5], anti-fogging [6], antiicing [7], energy conversation [8], self-cleaning [9,10], anti-corrosion [11,12], and friction drag reduction [13], etc. In general, there are two key points to equip surfaces with superhydrophobicity: creating a suitable surface roughness with micro-nanoscale hierarchical structures and low surface energy material modifications, which derived from the phenomenon of superhydrophobicity in nature $[14,15]$. Therefore, the combination of appropriate surface roughness and materials with low surface energy is a successful way to prepare superhydrophobic surfaces, for instance, advanced materials including carbon nanotubes [16,17], carbon nanofibers [18,19], graphene [20], graphene oxide [21] and so on. A mong these nanomaterials, Carbon nanotubes (CNTs) have attracted much attention in fabricating films because of their intrinsic 1-dimensional pore structure and unusual chemical and physical properties, including high surface area, good lubricity, hardness and toughness for superior transport capability $[22,23]$. Since carbon nanotubes were fist observed by lijima in 1991, a variety of them have become available, such as single, double, few, and multi-walled carbon nanotubes (MWCNTs) $[24,25]$. Besides, the MWCNTs are more active than single-walled carbon nanotubes because of it contain a large amount of hydroxyl groups. It was reported that the presence of hydroxyl groups could enhance the superhydrophobic properties $[26,27]$.

$\mathrm{Li}$ et al. synthesized a superhydrophobic bionic surface, this bionic surfaces were fabricated by depositing CNTs on PS colloidal crystals by the wet chemical self-assembling technique and the subsequent chemical treatment of the surface with fluoroalkylsilane [16]. Losic et al. prepared 3D graphene-carbon nanotube aerogels via a reaction, which exhibit superior adsorption 
performance [28]. Mokarian et al. fabricated a superhydrophobic composite comprising multi-walled carbon nanotubes and silicone rubber by etching, the contact angle of de-ionized water droplets on the prepared surface was measured with the larger value [29]. As a result, MWCNTs could be a promising candidate to effectively improve the micro-nanoscale hierarchical structures so as to increase the surface roughness, which should be conducive to the formation of superhydrophobicity. However, most of those methods could include severe conditions, such as expensive materials, strict deposition conditions and complex multi-step processing, and those could limit its practical application immensely. In contrast, electrochemical deposition, having the benefits of facile operation, moderate costs and reproducibility, is a more appropriate technique to produce water-repellent surfaces for engineering applications. At present, our group has obtained a compact superhydrophobic carbon-based film via the incorporation of cobalt by simple one-step high-voltage electrochemical deposition method, and this method presents a simple, efficient and less energy-consuming features. Cobalt has good hardness, strength and wear resistance. The dispersion of cobalt particles into the amorphous carbon matrix can not only relieve the internal stress of the film, but also effectively prevent the agglomeration and oxidation of the nano-metal particles, and the cobalt particles can form a layered micronanostructure on the surface of diamond-like carbon film. Based on previous works, the M WCNTs was used to enhance the superhydrophobicity of as-fabricated film with much better mechanical properties, self-cleaning and corrosion resistance.

In this study, a novel but simple path for fabricating multi-walled carbon nanotubes enhanced superhydrophobic MWCNTs-Co/a-C:H carbon-based film has been demonstrated via a safe technology, namely one-step electrochemical deposition. This process was adapted to use a MWCNTs/cobalt (II) acetylacetonate methanol solution as electrolyte without using aqueous solution to produce nanocomposite superhydrophobic films on silicon slice. For all we know, this kind of superhydrophobic ternary MWCNTs-Co/a-C:H carbon-based film with fairly strong mechanical strength and excellent anticorrosion and selfcleaning properties haven't yet been 
reported. The M WCNTs and cobalt nano-particle inserts in amorphous carbon were effective to tailor the feature of nanocrystallite/amorphous microstructure as well as micronanoscale hierarchical surface in favor of superhydrophobicity of M WCNTs-Co/a-C:H film with the contact angle of $158.1^{\circ}$. and sliding angle of $2.98^{\circ}$. without any further chemical modification. This new outstanding robust superhydrophobic MWCNTs-Co/a-C:H carbon-based film with excellent self-cleaning and corrosion resistance could have potential applications in the industry.

\section{Experimental procedure}

2.1 Materials and reagents

Multi-walled carbon nanotubes (MWCNTs, purity e95\%) were purchased from Kefu nano-materials Tech Co., Ltd, Nanjing, China, and the average diameter and average length of a M W CNT was 20-30 nm and 10-30 mm, respectively. A nalytical pure methanol was provided by Zhenxing chemical plant, Shanghai, China. Cobalt (II) acetylacetonate was supplied by Alfa Aesar, China. All of the above reagents were analytical reagent grades.

\subsection{Preparation of electrolyte solution}

The MWCNTs with $100 \mathrm{mg}$ were dispersed in $1 \mathrm{~L}$ methanol, then sonication was used to facilitate dispersion for $48 \mathrm{~h}$. Secondly, $100 \mathrm{mg}$ cobalt (II) acetylacetonate was dissolved in $500 \mathrm{~mL}$ methanol, likewise, sonication was used to facilitate dissolution for $10 \mathrm{~min}$. Finally, the electrolyte solution was consist of $50 \mathrm{~mL} 0.2 \mathrm{~g} / \mathrm{L}$ cobalt (II) acetylacetonate/methanol solution, $0.7 \mathrm{~mL} 0.1 \mathrm{~g} / \mathrm{L}$ MWCNTs/methanol suspension, and 49.3 $\mathrm{mL}$ analytical pure methanol solution. 


\subsection{Substrate processing}

M onocrystalline silicon wafers with dimensions of $0.7 \mathrm{~mm} \times 15 \mathrm{~mm} \times 30 \mathrm{~mm}$ was used to as a substrate. All of $\mathrm{Si}$ the substrates were cleaned using ultrasonic in methanol for $5 \mathrm{~min}$ and then immersed in aqueous solution of $10 \% \mathrm{HF}$ for $5 \mathrm{~min}$ to remove the native oxide layer. Finally, the Si substrates were ultrasonic cleaning with methanol for 5 min again.

Preparation of M WCNTs-Co/a-C:H nanocomposite film

The MWCNTs/cobalt nanocomposite superhydrophobic film was fabricated successfully on Si substrate via a green and safe one-step electrochemical deposition under high voltage, atmospheric pressure and low temperature. The dimensions of 0.7 $\mathrm{mm} \times 15 \mathrm{~mm} \times 30 \mathrm{~mm} \mathrm{Si}$ substrate was employed as the cathode and the same dimensions platinum tablets was employed as the anode, and the distance between two electrodes was $8 \mathrm{~mm}$. In order to ensure the accuracy of the experimental process, this research was strict to control the following conditions: the electrolytic temperature was controlled in the water bath of the constant temperature of $55^{\circ}$; the time of passing the inert gas and the process of deposition were controlled in 8 hours; and the voltage was set to $1200 \mathrm{~V}$.

In addition, the preparation method and deposition conditions of the pure diamond-like carbon (DLC) film were consistent with that of MWCNTs/cobalt nanocomposite film, but the electrolyte was only methanol.

\subsection{Characterization}


The morphology of MWCNTs-Co/a-C:H film was observed by a field emission scanning electron microscope (SEM) (MLA650F, FEI, USA). The scanning probe microscope (SPM) (Veeco Dimension 3100) was used to characterize the surface roughness of as-prepared film. The microstructures of the as-prepared film was further characterized by using high-resolution transmission electron microscope (HRTEM) (FEI Tecnai G2-20). X-ray photoelectron spectroscopy (XPS) was applied to analyze the chemical composition of as-prepared film on an AXIS ULTRA apparatus (Shimadzu-K ratos, Japan). In addition, all water contact angle and sliding angle was measured at ambient temperature using a measuring apparatus (DCAT21, Germany). The value of water contact angle was the average measurement of 5 different positions on the same sample surface.

A corrosion monitoring instrument (CHI660E, China) was used to observe the electrochemical corrosion of the film. The saturated potassium chloride $(\mathrm{KCl})$ electrode was used as a reference electrode with a platinum plate as the counter electrode. The as-prepared film was used as the working electrode and the exposed area was controlled at $1 \pm 0.1 \mathrm{~cm}^{2}$. The measurements were performed in $3.5 \% \mathrm{wt} . \mathrm{NaCl}$ solution at ambient temperature.

\section{Results}

\subsection{Surface morphology and wettability}

The surface morphology of pure DLC film and MWCNTs-Co/a-C:H film were examined by field emission scanning electron microscopy (SEM). SEM images of the 
surface of pure DLC film in different magnifications are shown in Fig. $1(a, b)$. It can be seen that the surface morphology of pure DLC film was very smooth, even at high magnification (Fig. 1 (b)) can only see the tiny protrusions. During electrochemical deposition, methanol electrolysis was accompanied by the generation of hydrogen, hydrogen has a cavitation effect on the surface of the film to form tiny protrusions.

SEM images of the surface of MWCNTs-Co/a-C:H film in different magnifications are displayed in Fig. 1 (c-f). As shown in Fig. 1 (c), with the incorporation of cobalt and MW CNTs, the surface roughness of MWCNTs-Co/a-C:H film sharply increases. M ore importantly, cobalt nano-particles and MWCNTs were embedded into the amorphous carbon films. This is more evident at higher magnification (Fig. 1 (d)). The MWCNTs grow up through cobalt nano-particles and exhibit a micro-nanoscale hierarchical structure. Further amplification found the morphology of MWCNTs (Fig. 1 (e)) and cobalt nano-particles (Fig. 1 (f)) are protruding structure. Especially, the structure of cobalt nano-particles was a pine-cone-like hierarchical micro-nanostructure. In general, this hierarchical micro-nanostructure could prompted the film surfaces to obtain a larger contact angle and smaller sliding angle in previous research [27]. Because the hierarchical micro-nanostructure can keeps the air underneath a water droplet on the surface. 


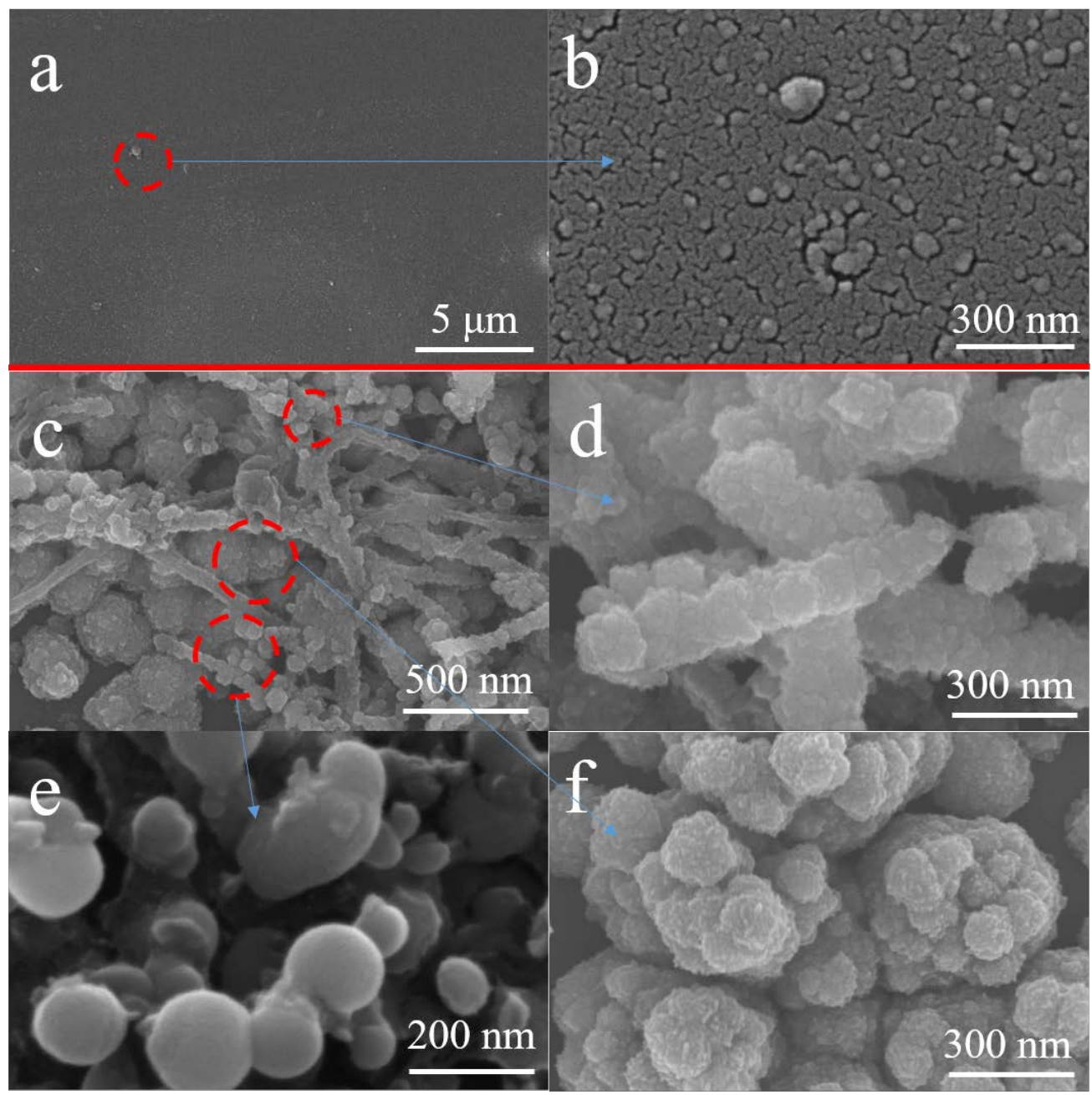

Fig. 1 Surface morphology of pure DLC film (a, b) and M WCNTs-Co/a-C:H film (c-f)

Typical data set of SPM images $\left(10 \times 10 \mu \mathrm{m}^{2}\right)$ of pure DLC film and M W CNTs-Co/a-C:H film are shown in Fig. 2 (a) and (b), respectively. The topography image of pure DLC film shows a surface formed by globular particles with, in average, diameters smaller than $200 \mathrm{~nm}$. Furthermore, the average roughness ( $\mathrm{Ra}$ ) of pure DLC film was $36.6 \mathrm{~nm}$ with relatively smooth surface and small grains. Compared to SEM, the SPM 2D projections images showed similar morphology. As shown in Fig. 2 (b), the M W CNTs-Co/a-C:H film was formed from spherical particles with an average diameter 
about $400 \mathrm{~nm}$. In addition, the average roughness (Ra) of MWCNTs-Co/a-C:H film is about $152 \mathrm{~nm}$, and noticeable protrusions were observed on the surface. The SPM 2D topography image show a similar morphology compared to SEM, also. It is indicated that the incorporation of cobalt and MWCNTs increases the roughness of the M W CNTs-Co/a-C:H film. This is an important factor for promoting the formation of superhydrophobic films.

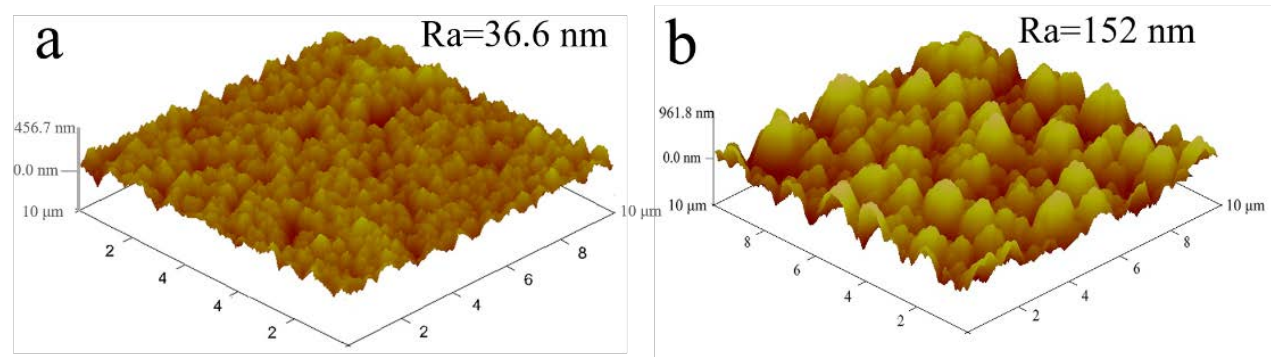

Fig. 2 SPM images of pure DLC film (a) and M WCNTs-Co/a-C:H film (b)

\subsection{Structural characterization}

X-ray photoelectron spectroscopy (XPS) was performed to analyse the chemical composition of pure DLC film and MWCNTs-Co/a-C:H film. The XPS spectra of pure DLC film mainly including $\mathrm{C} 1 \mathrm{~s}$ and $01 \mathrm{~s}$. Differently, the XPS spectra of MWCNTs-Co/a-C:H film mainly including $\mathrm{C} 1 \mathrm{~s}, 01 \mathrm{~s}$, and Co2p. M oreover, the peak position of Co2p is relatively sharp and the content of oxygen in the M WCNTs-Co/a-C:H film is more than that in the pure DLC film. This is because the M WCNTs surface contains a large number of oxygen-containing functional groups. It was also indicated that cobalt nano-particles and M WCNTs are successfully embedded into the amorphous carbon film. Furthermore, the $\mathrm{C} 1$ s of pure DLC film was fitted in the Gaussian mode including sp2C ]C (284.3 eV), sp3CeC (285.4 eV), and CeO or C JO (288.4 eV), as shown in Fig. 3(a). Similarly, there were four peaks presented in curve-fitted C1s spectra of MWCNTs-Co/a-C:H film, including sp2C]C (284.3 eV), sp3C-C (285.1 eV), MWCNTs C]C (285.6 eV), and $\mathrm{CeO}$ or C $] 0$ (288.5 eV) (Fig. 3(b)), which different with the C1s of pure DLC 
film due to the incorporation of MWCNTs formed MWCNTS C]C. Simultaneously, the incorporation of M WCNTs increases the oxygen content in the M W CNTs-Co/a-C:H film because the surface of the M W CNT s contains a large amount of oxygen-containing functional groups. It can provide an evidence for the superhydrophobic properties of MWCNTs-Co/a-C:H film. As shown in Fig. 3(c), the Co2p was fitted in the Gaussian mode including C02p3/2 (778.24 eV) and Co2p1/2 (793.34 eV). Comparison XPS standard spectrum, it can be seen that the film in the electron binding energy displacement are basically consistent with the elemental metal Co. This is consistent with TEM 's conclusion.

The structure of the M WCNTs-Co/a-C:H film was further investigated by TEM and HRTEM . Fig. 4(a) shown the TEM images of MWCNTs-Co/a-C:H film at low magnification. It is obvious that the cobalt nano-particles and MWCNTs are together in the carbon film, which indicating the cobalt nano-particles and M W CNTs were successfully embedded into the amorphous carbon film. At higher magnification (Fig. 4(b)), there were observed two different sizes of lattice fringes in M WCNTs-Co/a-C:H film. For further calculations, the d-spacing of the two lattice fringes with different sizes are $0.34 \mathrm{~nm}$ (Fig. 4(c)) and $0.18 \mathrm{~nm}$ (Fig. 4(d)), corresponding to (002) crystal plane of M W CNTs and (200) crystal plane of face-centered-cubic cobalt, respectively. It reveals that cobalt exists in the amorphous carbon film as an elementary substance.

The diffraction pattern and rings were shown in the electronic diffraction analysis through the corresponding selected area electron diffraction (SAED) pattern of MW CNTs-Co/a-C:H film, as shown in Fig. 5. There are obvious diffraction ring and many bright diffraction spots in the image of SAED. The diffraction ring is the diffraction pattern of elemental cobalt and there diffraction spots may be diffraction patterns of MWCNTs. Moreover, spherical Co particles have been further determined from the $d$-spacing (according to the Spacing $(n m)=L / R X$ ) evaluated of corresponding diffraction ring; the values of lattice fringes were $0.20 \mathrm{~nm}, 0.18 \mathrm{~nm}, 0.13 \mathrm{~nm}$, and $0.11 \mathrm{~nm}$, respectively corresponding to the crystalline face: $(111),(200),(220)$, and (311). It is 
demonstrated that the Co nano-particles and MWCNTs were inlaid together and successfully embedded into the MWCNTs-Co/a-C:H film with typical anocrystallite/amorphous microstructure.
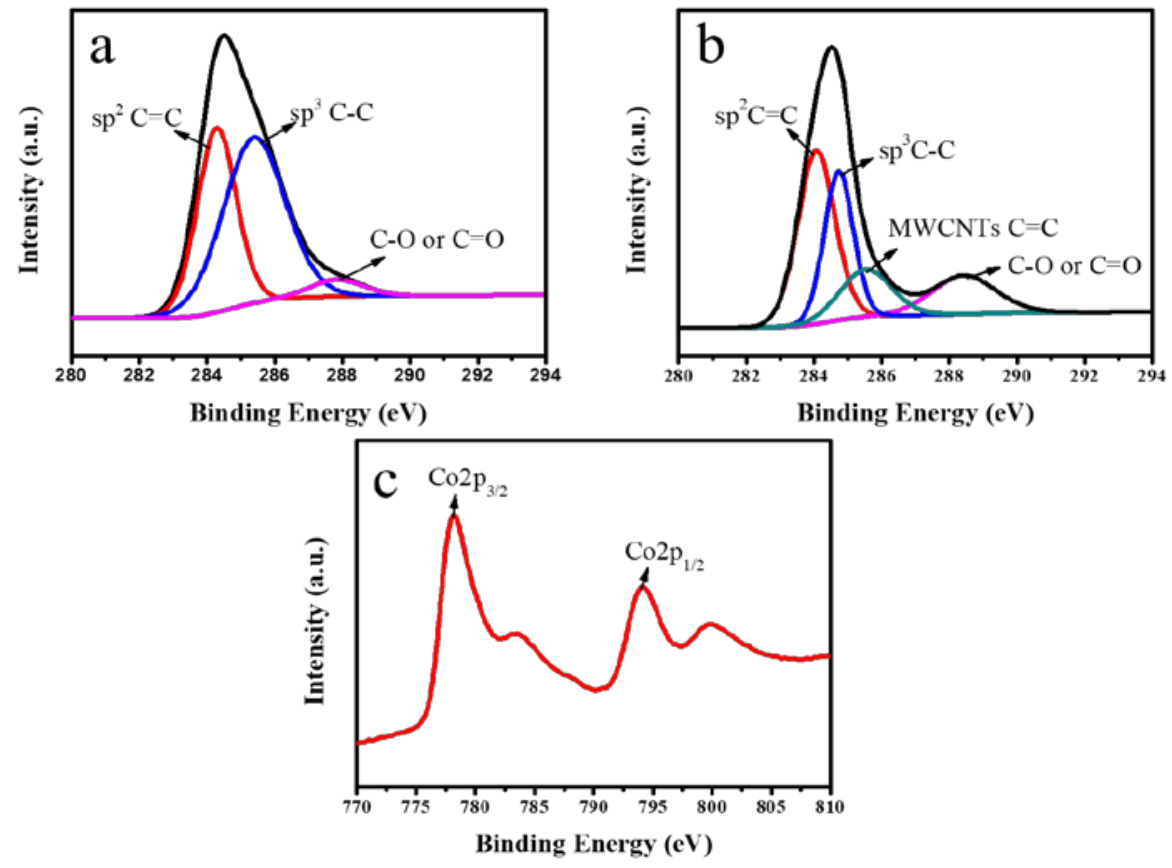

Fig. 3 C1s spectra of pure DLC film (a) and M WCNTs-Co/a-C:H film (b), Co2p spectra of

M W CNTs-Co/a-C:H film (c)

The structure of the M WCNTs-Co/a-C:H film was further investigated by TEM and HRTEM. Figure 4 (a) shows the TEM images of MWCNTs-Co/a-C:H film at low magnification. It is obvious that the cobalt nano-particles and M W CNTs are together in the carbon film, which indicating the cobalt nano-particles and MWCNTs were successfully embedded into the amorphous carbon film. At higher magnification (Fig. 4 (b)), there were observed two different sizes of lattice fringes in MWCNTs-Co/a-C:H film. For further calculations, the d-spacing of the two lattice fringes with different sizes are $0.34 \mathrm{~nm}$ (Fig. $4(\mathrm{c}))$ and $0.18 \mathrm{~nm}$ (Fig. $4(\mathrm{~d})$ ), respectively corresponding to $\left(\begin{array}{lll}0 & 0 & 2\end{array}\right)$ 
interplanar distance of M WCNTs and $\left(\begin{array}{lll}2 & 0 & 0\end{array}\right)$ interplanar distance of face-centered-cubic cobalt. It reveal that cobalt exists in the amorphous carbon film as an elementary substance.
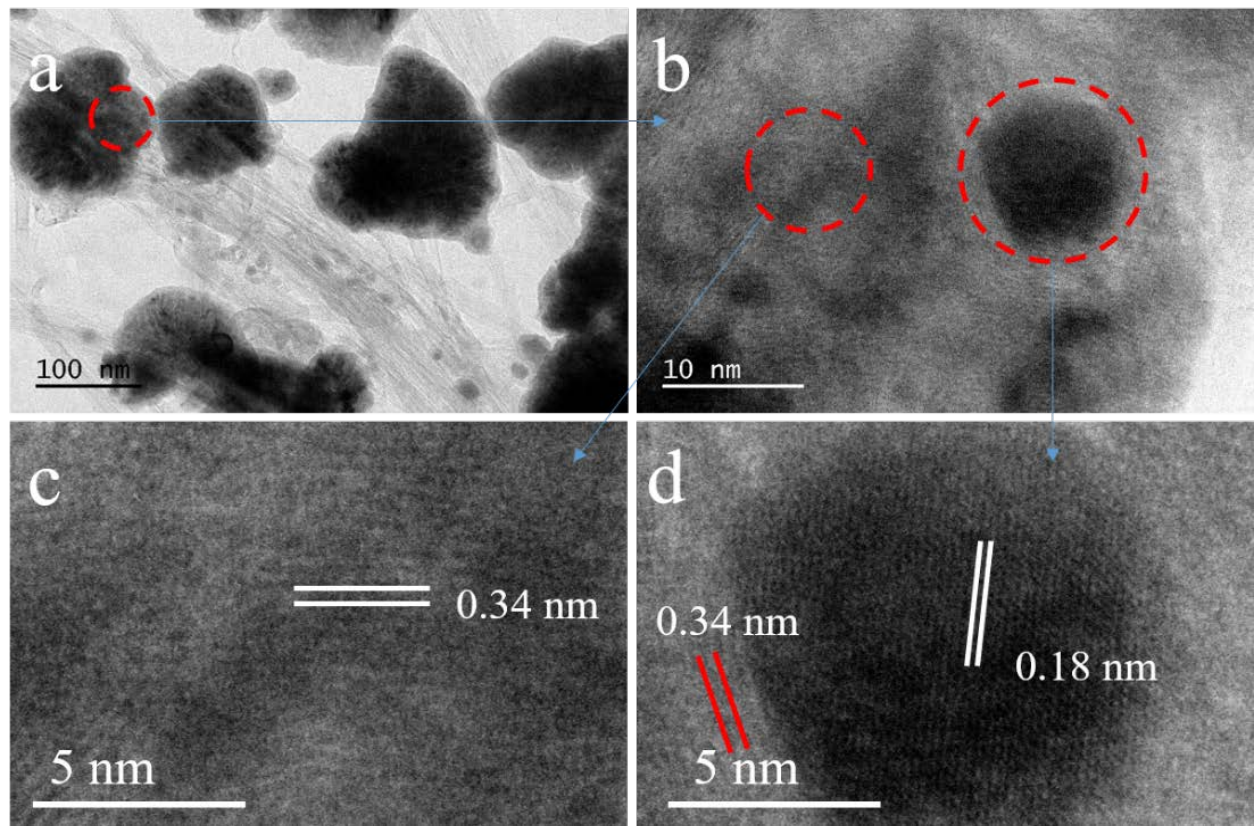

Fig. 4 TEM images of M W CNTs-Co/a-C:H film at different magnification (a-d)

The diffraction pattern and rings were shown in the electronic diffraction analysis through the corresponding selected area electron diffraction (SAED) pattern of MW CNTs-Co/a-C:H film, as shown in Fig. 5. There are obvious diffraction ring and many bright diffraction spots in the image of SAED. The diffraction ring is the diffraction pattern of elemental cobalt and there diffraction spots may be diffraction patterns of MWCNTs. M oreover, spherical Co particles have been further determined from the $d$-spacing (according to the Spacing $(n m)=L / R X$ ) evaluated of corresponding diffraction ring; the values of lattice fringes were $0.20 \mathrm{~nm}, 0.18 \mathrm{~nm}, 0.13 \mathrm{~nm}$, and 0.11

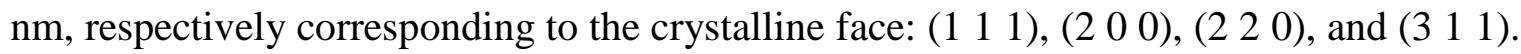


It is demonstrated that the Co nano-particles and MWCNTs were inlaid together and successfully embedded into the MWCNTs-Co/a-C:H film with typical nanocrystallite/amorphous microstructure.

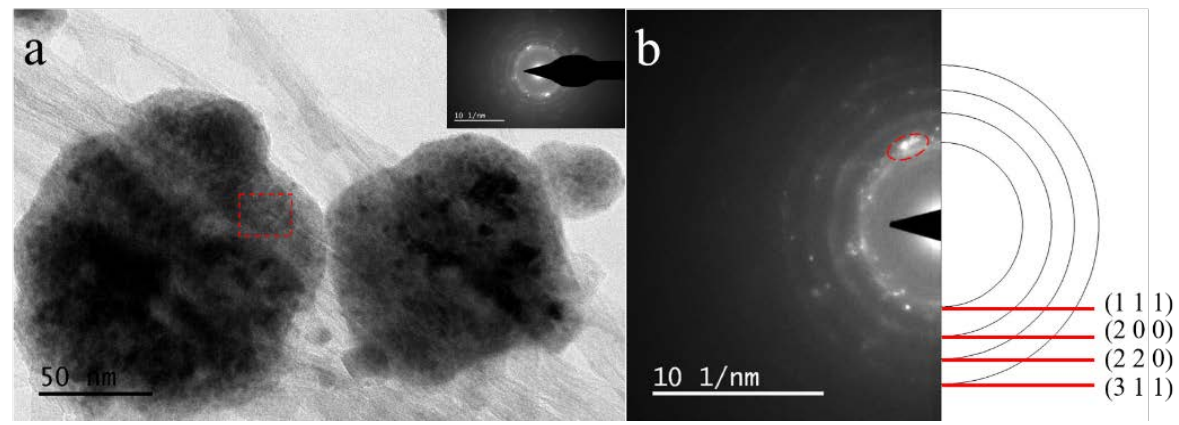

Fig. 5 SAED pattern of M WCNTs-Co/a-C:H film

\subsection{Wettability}

The wettability of films is mainly affected by two parameters of surface roughness and surface energy [31]. The wettability of the pure DLC film and the MWCNTs-Co/a-C:H film were evaluated by contact angle measurement with $5 \mu \mathrm{L}$ deionized water. The pure DLC film has a contact angle (CA) of $76.2^{\circ}$. due to its high surface energy (Fig. $6(\mathrm{~b})$ ). MWCNTs in the film is the main substances that reduce the surface energy of the MWCNTs-Co/a-C:H film because of the lower surface energy of the M WCNTs. On the other hand, the incorporation of M WCNTs and cobalt will increase the roughness of the film. The test results show that the highest contact angle of $158.1^{\circ}$. (Fig. $6(\mathrm{~d})$ ) and a sliding angle (SA) is approximately $2.98^{\circ}$. (Fig. $6(\mathrm{e})$ ) of the M WCNT s-Co/a-C:H film, which shows superhydrophobicity. According to Watanabe et al.'s report [32], the sliding angle may be affected by roughness, surface energy, and the mass of droplet. The high water contact angle and the low sliding angle indicate that the water droplet can easily roll down the surface when it tilts, which is attributable to the "lotus effect"[33]. The superhydrophobicity is greatly improved compared to pure DLC film. 


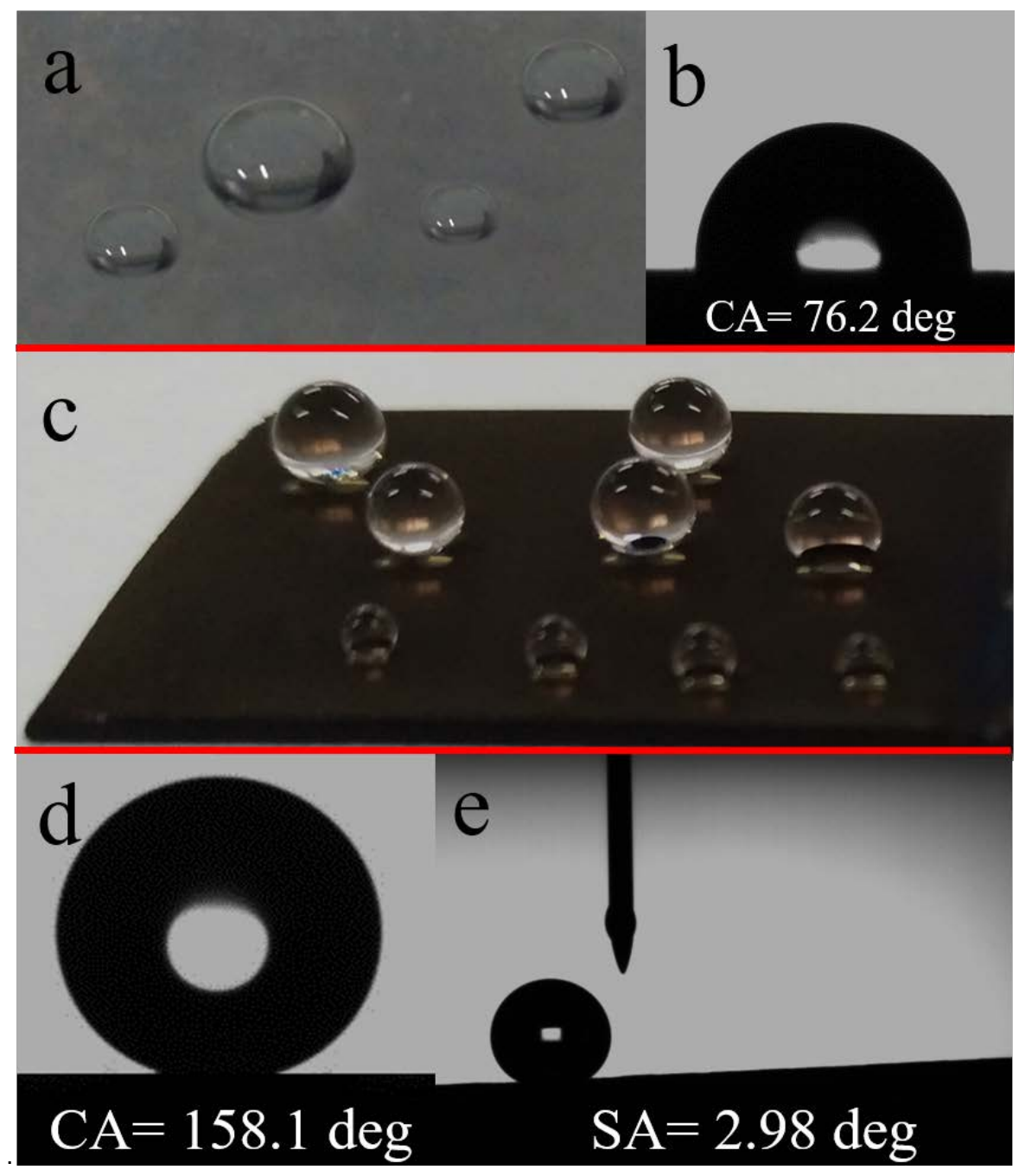

Fig. 6 Photographs of droplets on the pure DLC film (a), water contact angle of pure DLC film (b), photographs of droplets on the M WCNTs-CO/a-C:H film (c), water contact angle (d) and sliding angle (e) of M WCNTs-Co/a-C:H film

\subsection{Tape adhesive tests}

For the further study on robustness of painted surfaces, the tape peel test process and sandpaper abrasion test process of MW CNTs-Co/a-C:H film was shown in Figs. 7 and 8 [34-36]. Fig. 7(a) showed the superhydrophobicity of MWCNTs-Co/a-C:H film without adopting tape peel test, which exhibited the water contact angle of $158.1^{\circ}$. After 1 runs of tape test (Fig. $7(b)$ ), the WCNTs-Co/a-C:H film surface almost no change, still remained superhydrophobic and had a water contact angle of $156.9^{\circ}$. A fter 2 runs of tape test (Fig. 7(c)), the M W CNTs-Co/a-C:H film surface traces of some damage, the contact angle of $\mathrm{CNTs}-\mathrm{Co} / \mathrm{a}-\mathrm{C}: \mathrm{H}$ film is approximately 
$154.2^{\circ}$. Until 6 runs of tape test, the MWCNTs-Co/a-C:H film surface obvious signs of destruction, but the surface still remained superhydrophobic. Thus, due to the mechanical interlocking between MWCNTs and cobalt nano-particles, the superhydrophobic MWCNTS-Co/a-C:H nanocomposite film have fairly strong cohesion. However, the M W CNTs-Co/a-C:H film surface suffered serious damage after 8 runs of tape test and the contact angle of only $140.8^{\circ}$. Plot of tape test cycles and water contact angles after each tape peel test was shown in Fig. 7(g).

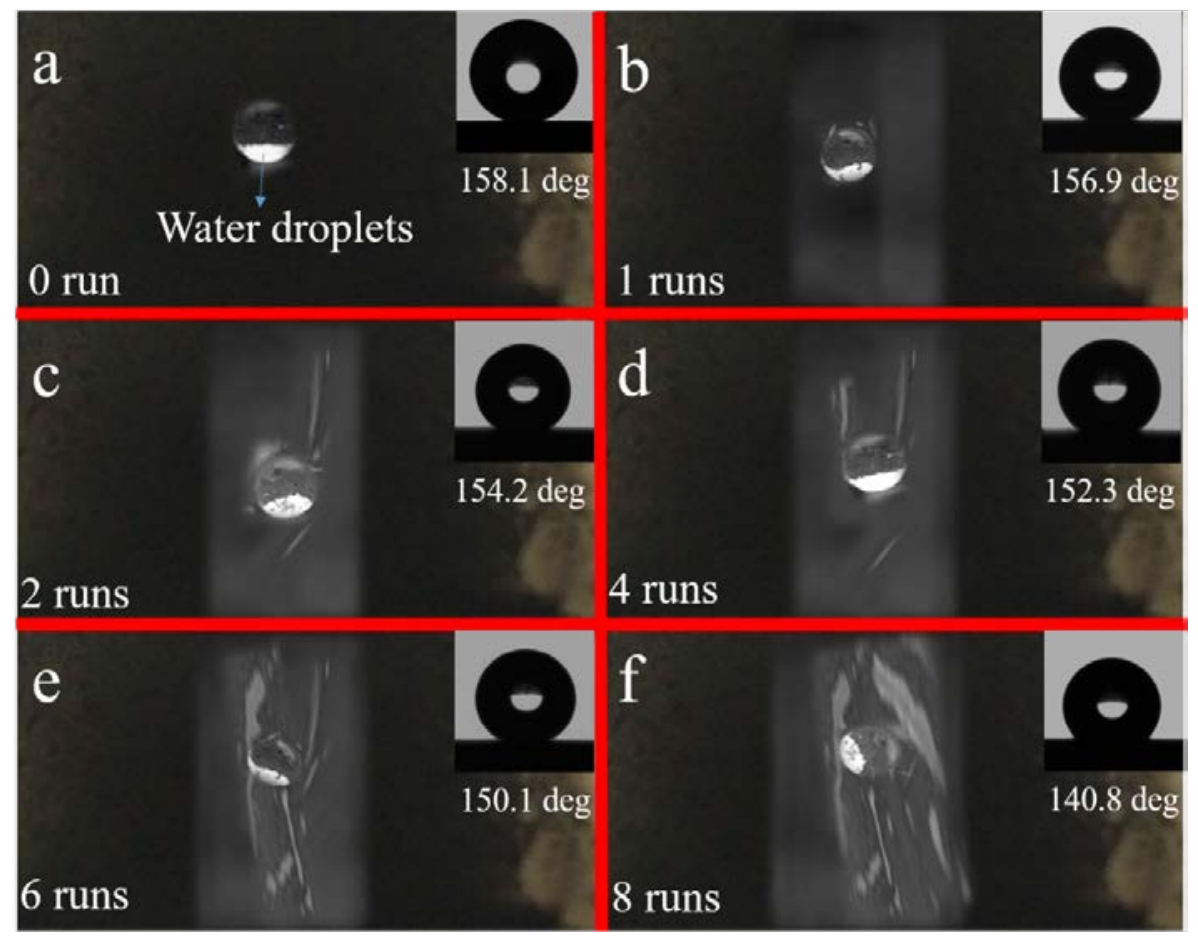

g

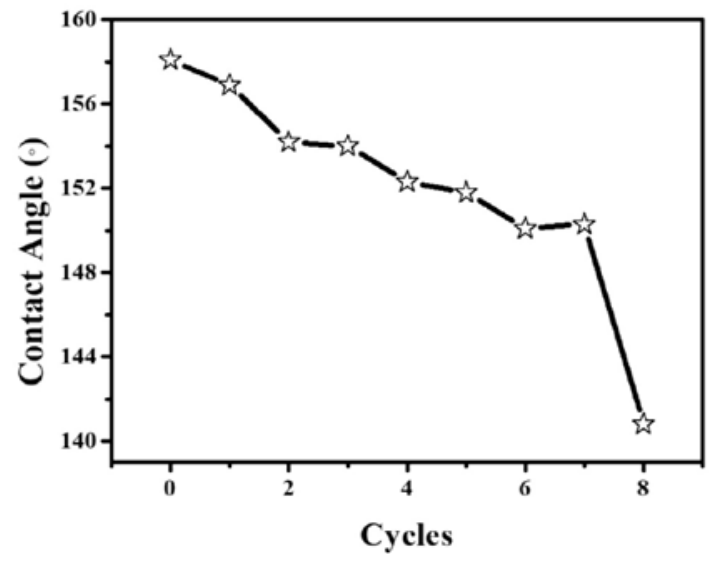

Fig. 7. Tape peel test process of MWCNTs-Co/a-C:H film (a-f) and plot of tape test cycles and water contact angles after each tape peel test $(\mathrm{g})$. 
Fig. 8 shows one cycle of the sandpaper abrasion test and plot of mechanical abrasion cycles and water contact angles after each abrasion test of MWCNTS-Co/a-C:H film. The M W CNT s-Co/a-C:H film was placed face-down to sandpaper under a weight at $100 \mathrm{~g}$ and moved longitudinally for $10 \mathrm{~cm}$ along the ruler (Fig. 8(a)). Then, the MWCNTs-Co/a-C:H film was rotated by $90^{\circ}$ in situ and then moved transversely for $10 \mathrm{~cm}$ along the ruler (Fig. 80(b)). This procedure guaranteed the surface was abraded both longitudinally and transversely, which could be defined as one abrasion cycle. A fter 20 cycles of sandpaper abrasion test, it can be observed that the superhydrophobic film was not peeled off from the substrates. M eanwhile, water contact angles after each abrasion cycle were shown in Fig. 8(c), and it can be seen that the static water contact angles were between $150^{\circ}$ and $158^{\circ}$, indicating the coated surface retained superhydrophobicity after sandpaper abrasion test. Again, the superhydrophobic WCNTS-Co/a-C:H film has strong mechanical property.

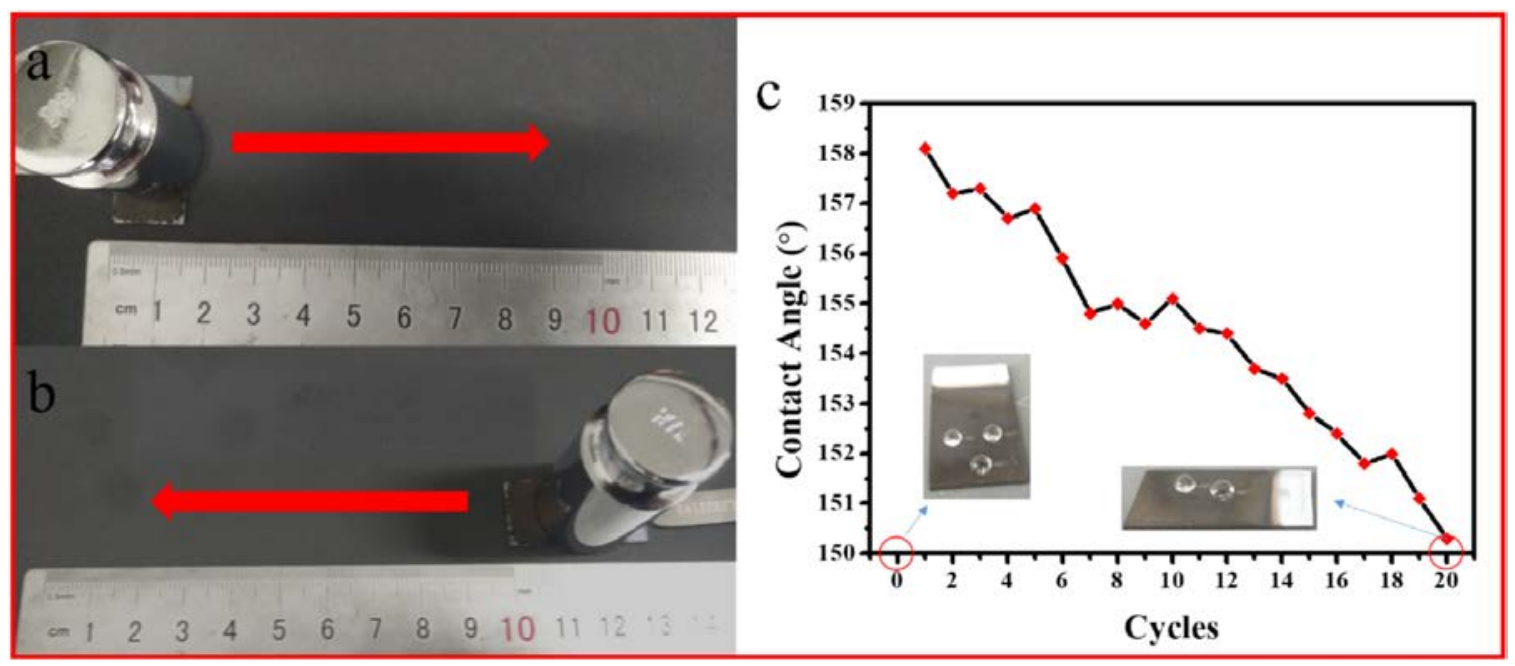

Fig. 8. O ne cycle of the sandpaper abrasion test $(a, b)$ and plot of mechanical abrasion cycles and water contact angles after each abrasion test (c).

\subsection{Corrosion resistance}

Electrochemical experiments can be used to assess the corrosion resistance of pure DLC film and MWCNTs-Co/a-C:H film. All samples were soaked in $3.5 \mathrm{wt} \% \mathrm{NaCl}$ 
aqueous solution for $2 \mathrm{~h}$ before testing to ensure that the samples were stable in the corrosive medium. Figure 9 showed the Tafel polarization curves for pure DLC film and MWCNTs-Co/a-C:H film. And the electrochemical parameters including corrosion potential $\left(E_{\text {corr }}\right)$, corrosion current density $\left(I_{\text {corr }}\right)$, and protection efficiency $(/ 7)$ of pure DLC film and MWCNTs-Co/a-C:H film based on the polarization curves was listed in Table 1. In general, the lower the corrosion current density, the lower the corrosion rate. Obviously, the corrosion current density of MWCNTs-Co/a-C:H film reached $4.234 \times 10^{-10} \mathrm{~A} \cdot \mathrm{cm}^{-2}$ and the corrosion potential up to $-0.268 \mathrm{~V}$. Compared to pure DLC film, the MWCNTs-Co/a-C:H film exhibit lower corrosion rate, indicating the incorporation of cobalt and MWCNTs in the deposit significantly enhanced the corrosion performance. M oreover, the protection efficiency (П) (Calculated according to Equation D) of MWCNTs-Co/a-C:H film is approximately $98.02 \%$. It revealed that the M W CNTs-Co/a-C:H film have outstanding corrosion resistance.

$\mathrm{I}^{0}$ corr is the corrosion current density of pure DLC film; $\mathrm{I}_{\text {corr }}$ is the corrosion current density of M WCNTs-C $\mathrm{O} / \mathrm{a}-\mathrm{C}: \mathrm{H}$ film. 


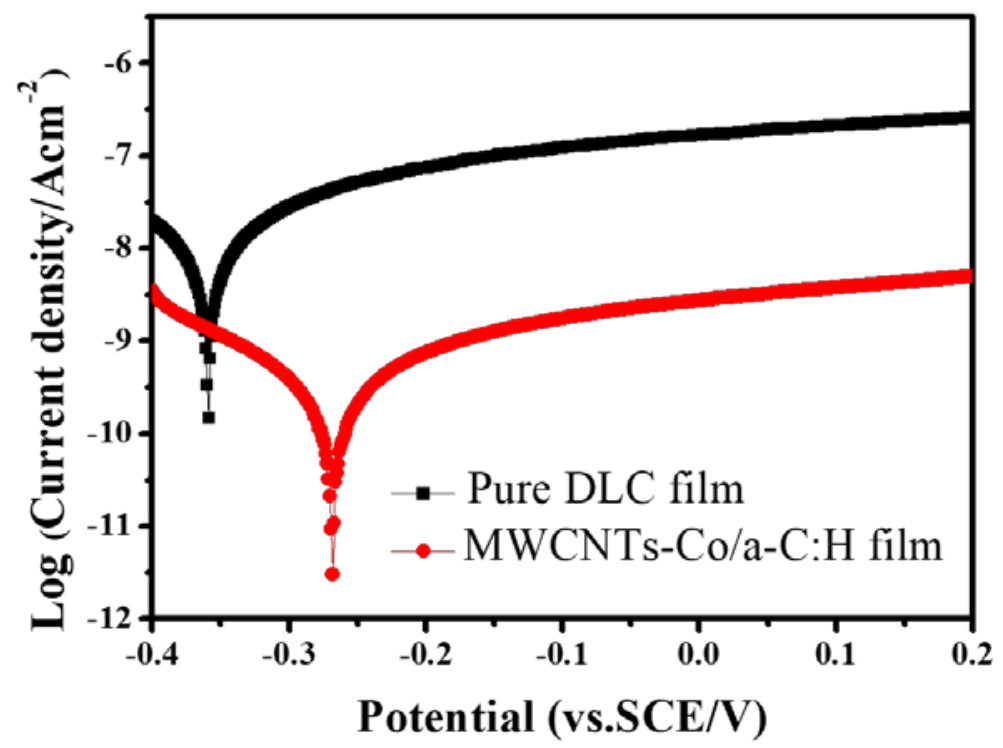

Fig. 9 Tafel polarization curves for pure DLC film and M WCNTs-Co/a-C:H film

Table 1 Electrochemical parameters for pure DLC film and M W CNTs-Co/a-C:H film obtained from polarization curves

\begin{tabular}{ccc}
\hline Samples & Pure DLC film & MWCNTs-Co/a-C:H film \\
\hline Icorr $\left(\mathrm{A} \cdot \mathrm{cm}^{-2}\right)$ & $2.141 \times 10^{-8}$ & $4.234 \times 10^{-10}$ \\
Ecorr (V) & -0.359 & -0.268 \\
$\eta(\%)$ & 98.02 \\
\hline
\end{tabular}

The electrochemical impedance spectroscopy (EIS) can be used to evaluate the protection of the films against corrosion. EIS plots were recorded over the frequency range from $0.01 \mathrm{~Hz}$ to $10^{5} \mathrm{~Hz}$ at open circuit potential in $3.5 \mathrm{wt} \% \mathrm{NaCl}$ aqueous solution as shown in Fig. 10 and Fig. 11. The Nyquist plots of pure DLC film and MW CNTs-Co/a-C:H film were shown in Fig. 10. Apparently, the radius of capacitive semi-circle of MWCNTs-Co/a-C:H film is much larger than the pure DLC film. In 
addition, in order to further study the anti-corrosion properties of the films, ZSimp-Win software can be used to analyze the impedance spectrum through the equival ent circuit simulation method. The equivalent circuit include capacitors, resistors and insulators, where $Q$ is the constant phase angle element, $Q_{\text {film }}$ represents the film capacitance, $Q_{d l}$ represents the electric double layer capacitance between the surface of the silicon pellet and seawater, and $\mathrm{n}$ is the constant phase angle index, indicating the degree of dispersion effect. $R_{s}$ is the solution resistance, $R_{\text {film }}$ is the film resistance, $R_{c t}$ is the charge transfer resistance. The equivalent circuit diagram of pure DLC film and MWCNTs-Co/a-C:H film was shown in Fig. 11 (b). It is worth mentioning that the $R_{\text {film }}$ of M W CNTs-Co/a-C:H film is about $2.01 \times 10^{6} \odot \cdot \mathrm{cm}^{2}$ bigger than the pure $\mathrm{DLC}$ film $\left(\mathrm{R}_{\text {film }}=\right.$ $\left.9.71 \times 10^{4} \odot \cdot \mathrm{cm}^{2}\right)$ and the $Q_{\text {film }}$ of MWCNTs-Co/a-C:H film is about $7.12 \times 10^{-7} \mathrm{~F} \cdot \mathrm{cm}^{2} \cdot$ In general, the impedance value is used to characterize the corrosion resistance of the film, which is generally considered a sign of the corrosion resistance of the film. The greater the value, the better the corrosion resistance of the film. Furthermore, the B ode plots of pure DLC film and MWCNTs-Co/a-C:H film was shown in Fig. 11 (a). Similarly, the impedance value of the low frequency region is used to characterize the corrosion resistance of the film. The greater the value, the better the corrosion resistance of the film. The Tafel polarization curves and electrochemical impedance spectroscopy indicated that the corrosion resistance behavior of M WCNTs-Co/a-C:H film is better than that of pure DLC film. In other words, the MWCNTs-Co/a-C:H film could present outstanding corrosion resistance, indicating some potential uses as anti-corrosion materials. 

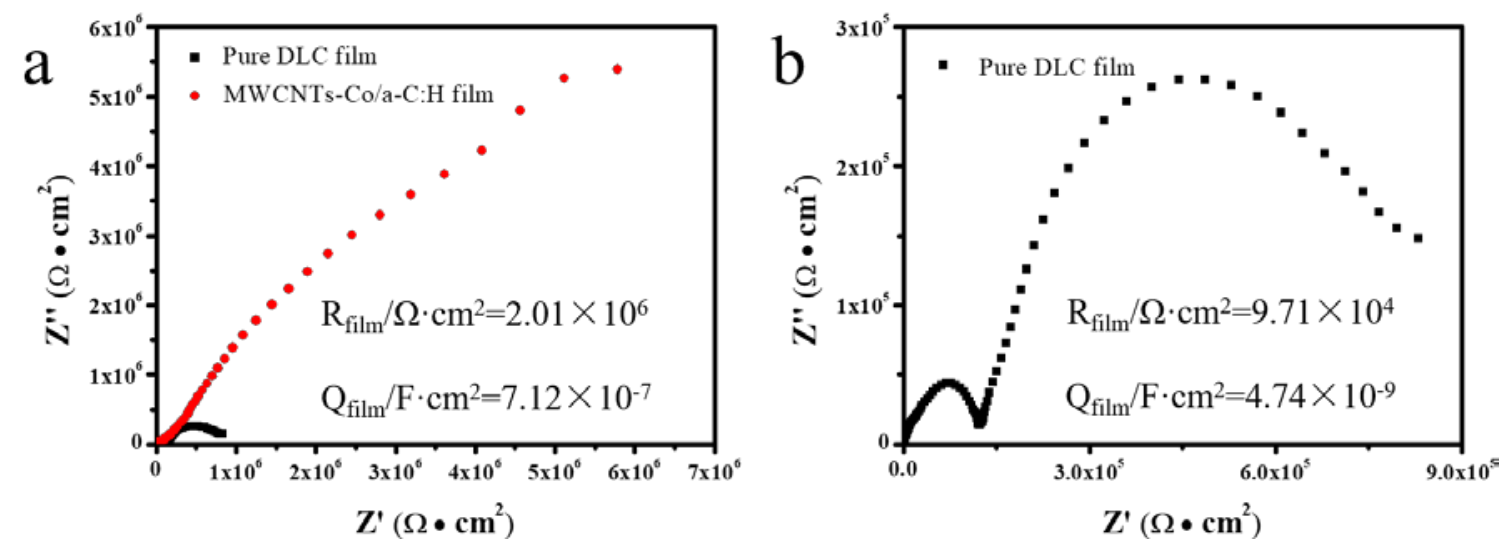

Fig. 10 N yquist plots of M W CNTs-Co/a-C:H film (a) and pure DLC film (b)
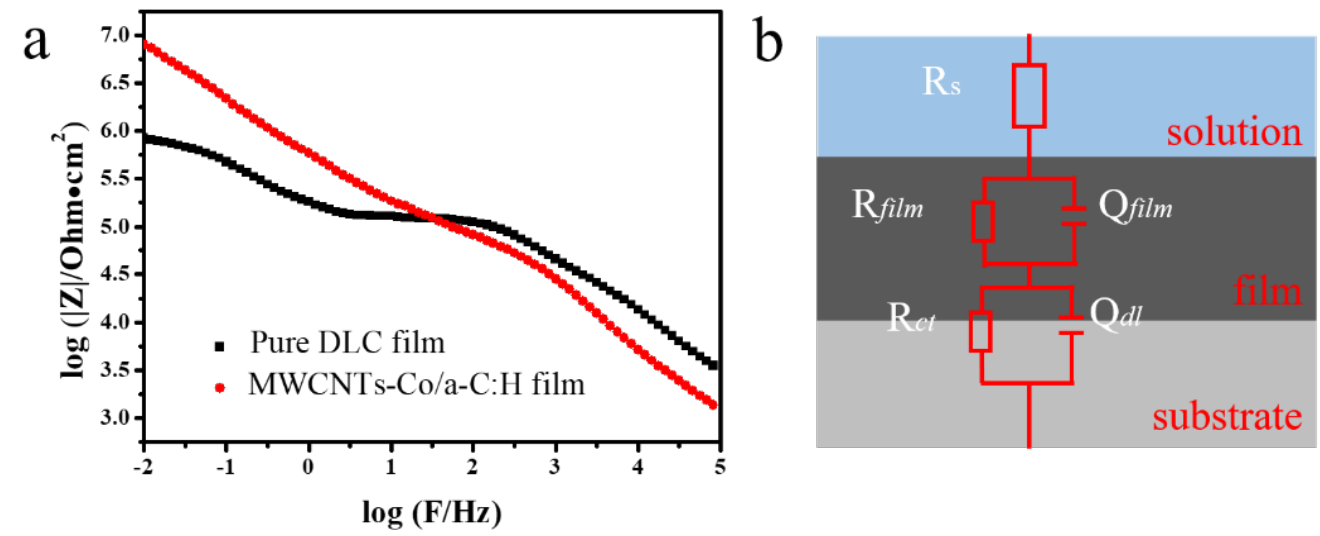

Fig. 11 Bode plots of pure DLC film and MW CNTs-Co/a-C:H film (a) and equivalent circuit for EIS data fitting (b)

\subsection{Self-cleaning}

It is important for superhydrophobic surfaces with self-cleaning ability for their practical applications. Fig. 12 (a-d) effectively illustrated the self-cleaning behavior process of pure DLC film and MWCNTs-Co/a-C:H film. The pure DLC film was placed on the left and the superhydrophobic MWCNTs-Co/a-C:H film was placed on the right side. First, all the films surface covered with red chalk dust (Fig. 12 (a)). Then, a few drops of $0.05 \mathrm{ml}$ deionized water in the pure DLC film and MWCNTs-Co/a-C:H film 
surface, respectively. It can be clearly seen that the water drops with red chalk dust quickly rolled down in the MWCNTs-Co/a-C:H film surface. More importantly, the MWCNTs-Co/a-C:H film became clean and restored the initial superhydrophobic properties. However, the water droplets remain stationary on the surface of pure DLC film, which not show the self-cleaning ability. Compared to pure DLC film, the M W CNTs-Co/a-C:H film exhibited excellent self-cleaning properties.

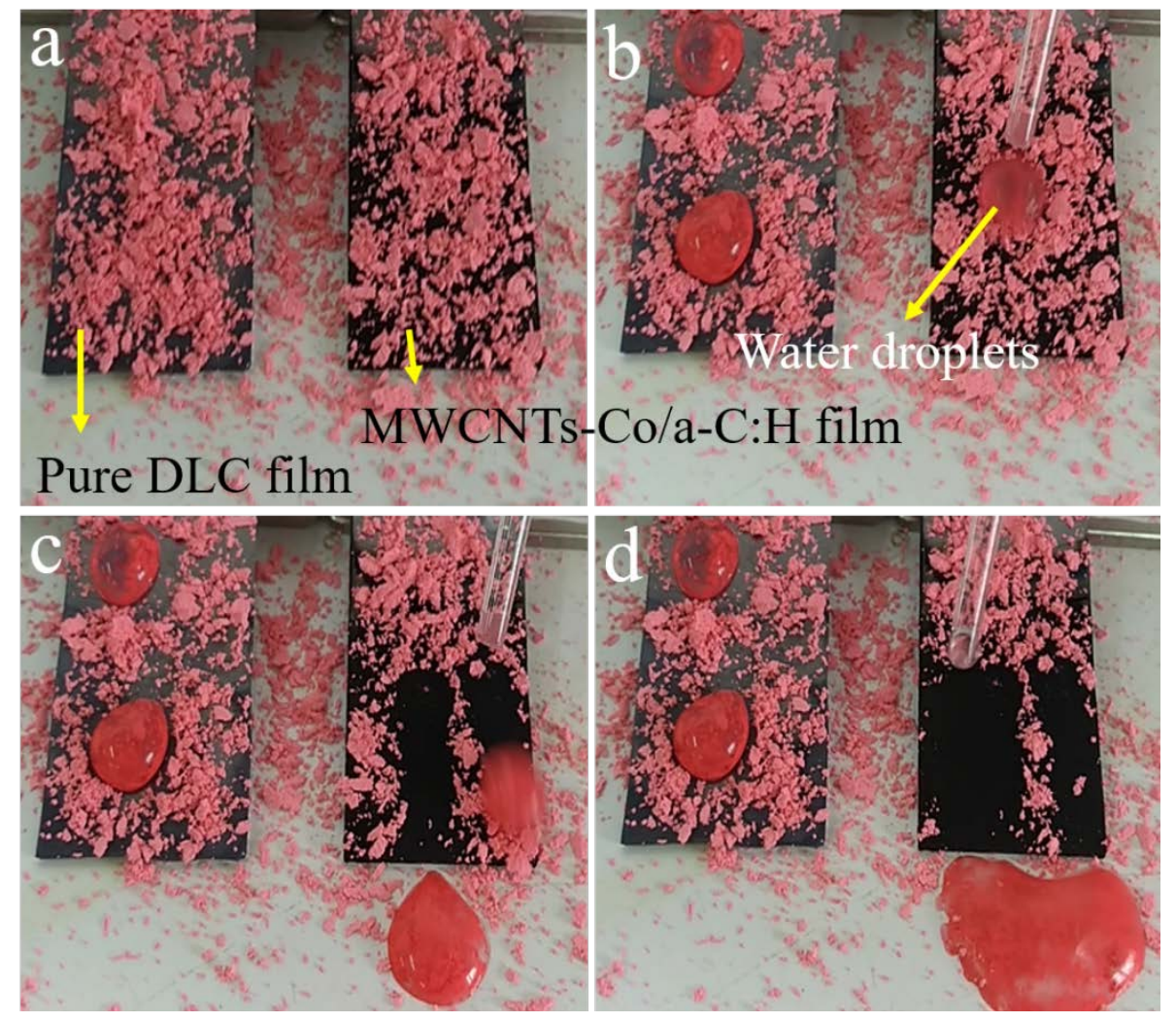

Fig. 12 Self-cleaning process (a-d) of M WCNTs-Co/a-C:H film

\section{Discussions}

In this study, a novel but simple path electrochemical deposition for the preparation of outstanding superhydrophobic M WCNTs-Co/a-C:H nanocomposite carbon-based film with fairly strong mechanical strength and anti-corrosion as well as self-cleaning 
performances. The water contact angle of MWCNTs-Co/a-C:H nanocomposite superhydrophobic film surface is approximately 158.1 deg and a sliding angle is about $2.98 \mathrm{deg}$, which due to the incorporation of M W CNTs and cobalt nano-particles resulting in the film to form a micro-nanoscale hierarchical structure. This micro-nanoscale hierarchical structure can effectively keep the air between the water droplets and the film surface, so that the water droplets on the film surface can quickly roll down, maintaining superhydrophobic properties. M oreover, the M W CNTs surface contains a large number of oxygen-containing functional groups could be another factor to obtain super-hydrophobic properties of the MWCNTs-Co/a-C:H film. Remarkably, the incorporation of MWCNTs can effectively reduce the surface energy of the M W CNTs-Co/a-C:H carbon-based film.

The formation mechanism of superhydrophobic M W CNTs-Co/a-C:H carbon-based film on the Si substrate through a green and safe one-step electrochemical deposition was exhibited in Fig. 13. When the power is turned on, first, methanol starts electrolysis into $\mathrm{CH}_{3}{ }^{+}$. In the meantime, cobalt (II) acetylacetonate was also electrolyzed into $\mathrm{CO}^{2+}$ and $\left(\mathrm{C}_{5} \mathrm{H}_{7} \mathrm{O}_{2}\right)^{-}$. Then, under the action of high voltage electric field, the $\mathrm{CH}_{3}{ }^{+}$and $\mathrm{CO}^{2+}$ drive the M W CNTs to the cathode. Finally, the M WCNTs-Co/a-C:H film was formed on the Si substrate. In this process, due to the strong conductivity of MWCNTs will promote the movement of $\mathrm{CH}_{3}{ }^{+}$and $\mathrm{CO}^{2+}$, accelerate the deposition process. The mechanism of this process is listed as follows:

$\mathrm{CH} 3 \mathrm{OH}^{\prime} \mathrm{CH} 3++\mathrm{OH}-$ 
$\mathrm{Co}(\mathrm{C} 5 \mathrm{H} 702) 2^{\prime} \mathrm{CO} 2++2(\mathrm{C} 5 \mathrm{H} 702)-$

$\mathrm{Co2}++\mathrm{CH}+$ MWCNTs' MWCNTs-Co/a-C: H film

As shown in Fig. 13, the superhydrophobic schematic illustration of M W CNTs-Co/a-C:H film was vividly demonstrated. First, cobalt (II) acetylacetonate and M W CNTs were electrolytically deposited on Si substrate in a $1200 \mathrm{~V}$ voltage and $55^{\circ} \mathrm{C}$ water bath. Then, the cobalt nano-particles and MWCNTs were inlaid grow on the surface to form a micro-nanoscale hierarchical structure. So, the superhydrophobicity of M W CNTs-Co/a-C:H film can be ascribed to the generation of a multi-scale hierarchical rough structure, which enables air pockets to be trapped in the cavities formed by the nano-features on the film surface, suspending the water droplets. The incorporation of MWCNTs not only increases the roughness of the film, but also reduces the surface energy of the film.
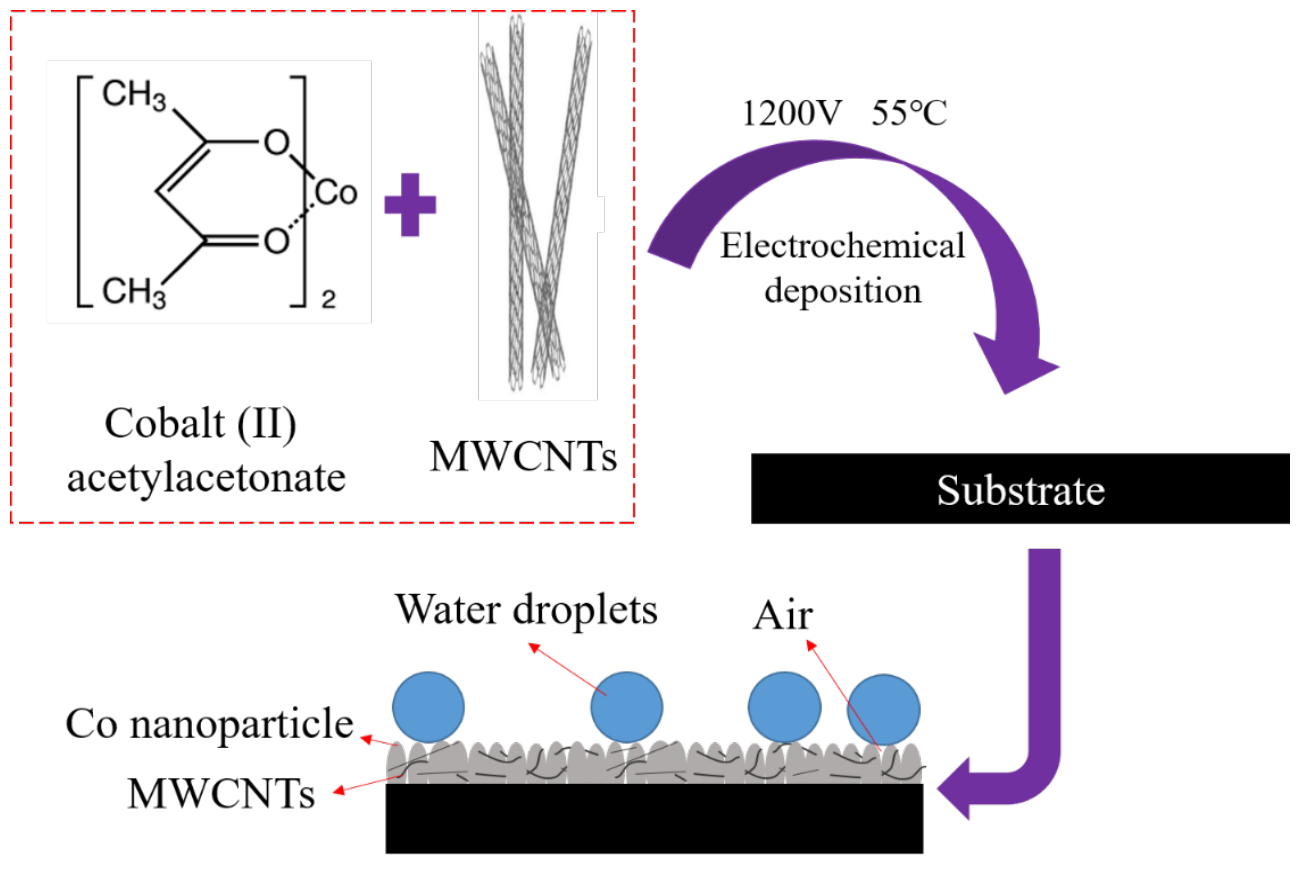

MWCNTs-Co/a-C:H film 
Fig. 13 Schematic of the preparation of superhydrophobic M WCNTs-Co/a-C:H film

The mechanism of corrosion resistance could probably be related to hydrophobicity of film. The change in corrosion performance might be similar to that of hydrophobicity. Due to the low wetted area on solid surface immersed in the aggressive solution, the trapped air between the clusters in the superhydrophobic surface can serve as an effective barrier to keep corrosive media away from the surface and provide better corrosion protection for the film. These results suggested that the superhydrophobic MW CNTs-Co/a-C:H film could achieve much better corrosion resistance than that of pure DLC film, which was attributed to the combined action of M W CNTs and nano-sized cobalt particles acting as barriers hinders the movement of $\mathrm{Cl}^{-}$in the $3.5 \%$ wt. $\mathrm{NaCl}$ solution. In other words, the MWCNTs-Co/a-C:H film have outstanding corrosion resistance.

The most important factor for self-cleaning phenomenon should be the incorporation of M WCNTs and nano-sized cobalt particles so as to form a micro-nanoscale hierarchical structure on the surface of the film. This micro-nanoscale hierarchical structure not only had a large micro-roughness, but also achieved a low surface energy for the M W CNTs-Co/a-C:H film duo to the presence of MWCNTs. Particularly, the air trapped in the space around the microstructures could reduce the contact area between the water droplet and the superhydrophobic surface, so that the water droplets could easily take away the dust on the super-hydrophobic film. As a result, the film could exhibit superior self-cleaning performance compared to the M WCNTs-Co/a-C:H film and pure DLC film, 
indicating that this kind of superhydrophobic MWCNTs-Co/a-C:H film might have potential applications in modern industry.

\section{Conclusions}

Multi-walled carbon nanotubes enhanced MWCNTs-Co/a-C:H carbon-based film was successfully fabricated via a green, safe, and one-step electrochemical deposition technology. Remarkably, the co-incorporation of MWCNTs and cobalt nano-particles could facilitate the formation of a micro-nanoscale hierarchical structure. The resulting MW CNTs-Co/a-C:H carbon-based film could exhibit outstanding superhydrophobicity with the contact angle of 158.1 deg and a sliding angle of 2.98 deg without any further surface modification meanwhile it could possess excellent anti-corrosion and self-cleaning performances. The as-fabricated surface could maintain superhydrophobicity after an adhesion tape test, which confirmed that the M WCNTs and cobalt nano-particles are fairly strong bond to the film. This research highlights a potential route for the industrial preparation of superhydrophobic film that has promising uses including self-learning, antifouling and anticorrosion applications such as ship hulls and aeroplane wings.

\section{Acknowledgements}

This work is supported by the National Natural Science F oundation of China (G rant No. 51611130190) and Program for Excellent Y oung Talents, JX UST (N o. JX UST QJ BJ 201611).

\section{R eferences}


[1] B. Wang, W. Liang, Z. Guo, W. Liu, B iomimetic super-lyophobic and super-lyophilic materials applied for oil/water separation: a new strategy beyond nature, Chem. Soc. Rev. 44 (2015) 336.

[2] Z. X u, Y. Zhao, H. W ang, H. Zhou, C. Qin, X. Wang, T. Lin, Fluorine-free superhydrophobic coatings with $\mathrm{pH}$-induced wettability transition for controllable oilwater separation, ACS A ppl. $M$ ater. Interfaces 8 (2016) 5661.

[3] J. Song, S. Huang, Y. L u, X. B u, J.E. M ates, A. Ghosh, R. Ganguly, C.J. Carmalt, I.P. Parkin, W. Xu, Self-driven one-step oil removal from oil spill on water via selective-wettability steel mesh, ACS A ppl. M ater. Interfaces 6 (2014) 19858.

[4] B. Chen, J. Qiu, E. Sakai, N. K anazawa, R. Liang, H. Feng, Robust and superhydrophobic surface modification by a "paint+adhesive" method: applications in self-cleaning after oil contamination and oil-water separation, A CS A ppl. M ater. Interfaces 8 (2016) 17659-17667. [5] L. Zhang, J. W u, M. Hedhili, X. Y ang, P. W ang, Inkjet printing for direct micropatterning of a superhydrophobic surface: toward biomimetic fog harvesting surfaces, J. M ater. Chem. A 3 (2015) 2844-2852.

[6] M. W en, L. W ang, M. Zhang, L. Jiang, Y . Zheng, A ntifogging and icing-delay properties of composite micro- and nanostructured surfaces, ACS A ppl. M ater. Interfaces 6 (2014) 3963-3968. [7] L. W ang, Q. Gong, S. Zhan, L. Jiang, Y. Zheng, Robust anti-icing performance of a flexible superhydrophobic surface, A dv. M ater. 28 (2016) 7729.

[8] X.F. Zhang, R.J. Chen, Y.H. Liu, J.M . Hu, Electrochemically generated sol-gel films as inhibitor containers of superhydrophobic surfaces for the active corrosion protection of metals, J. M ater. Chem. A 4 (2015) 649-656.

[9] Z. Y ang, L. Wang, W. Sun, S. Li, T. Zhu, W. Liu, G. Liu, Superhydrophobic epoxy coating modified by fluorographene used for anti-corrosion and self-cleaning, A ppl. Surf. Sci. 401 (2017).

[10] G. Zhao, Y. X ue, Y. Huang, Y . Y e, F.C. Walsh, J. Chen, S. Wang, One-step electrodeposition of a self-cleaning and corrosion resistant Ni/W S2 superhydrophobic surface, RSC A dv. 6 (2016) 439-443.

[11] F. Xiao, S. Y uan, B. Liang, G. Li, S. Pehkonen, T. Zhang, Superhydrophobic CuO nanoneedle-covered copper surfaces for anticorrosion, J. M ater. Chem. A 3 (2015) 4374-4388. [12] W. Liu, Q. X u, J. Han, X. Chen, Y. M in, A novel combination approach for the preparation of superhydrophobic surface on copper and the consequent corrosion resistance, Corros. Sci. 110 (2016) 105-113.

[13] M . Cheng, M. Song, H. Dong, F. Shi, Surface adhesive forces: a metric describing the drag-reducing effects of superhydrophobic coatings, Small 11 (2015) 1665. 
[14] Z. Guo, W. Liu, B.L. Su, Superhydrophobic surfaces: from natural to biomimetic to functional, J. Colloid Interface Sci. 353 (2011) 335.

[15] H. B ellanger, T. Darmanin, D.G.E. Taffin, F. Guittard, Chemical and physical pathways for the preparation of superoleophobic surfaces and related wetting theories, Chem. Rev. 114 (2014) 2694.

[16] Y . Li, X.J. Huang, S.H. Heo, C.C. Li, Y .K . Choi, W .P. Cai, S.O. Cho, Superhydrophobic bionic surfaces with hierarchical microsphere/SW CNT composite arrays, Langmuir 23 (2007) 2169.

[17] I.S. Bayer, A. Steele, E. Loth, Superhydrophobic and electroconductive carbon nanotube-fluorinated acrylic copolymer nanocomposites from emulsions, Chem. Eng. J . 221 (2013) 522-530.

[18] U. Cengiz, M .Z. A vci, H.Y . Erbil, A.S. Sarac, Superhydrophobic terpolymer nanofibers containing perfluoroethyl alkyl methacrylate by electrospinning, A ppl. Surf. Sci. 258 (2012) 5815-5821.

[19] A. Das, H.T. Hayvaci, M.K. Tiwari, I.S. Bayer, D. Erricolo, C.M . M egaridis, Superhydrophobic and conductive carbon nanofiber/PTFE composite coatings for EM I shielding, J. Colloid Interface Sci. 353 (2011) 311.

[20] L. X u, G. Xiao, C. Chen, R. Li, Y. M ai, G. Sun, D. Y an, Superhydrophobic and superoleophilic graphene aerogel prepared by facile chemical reduction, J. M ater. Chem. A 3 (2015) 7498-7504.

[21] S. Lu, H. Gao, Q. Wang, W. X u, S. Szunerits, R. B oukherroub, Fabrication of stable homogeneous superhydrophobic HDPE/graphene oxide surfaces on zinc substrates, RSC A dv. 6 (2016) 29823-29829.

[22] G. Hummer, J.C. Rasaiah, J.P. N ow oryta, W ater conduction through the hydrophobic channel of a carbon nanotube, N ature 414 (2001) 188.

[23] L. Dai, D.W . Chang, J.B. Baek, W . L u, Carbon nanomaterials for advanced energy conversion and storage, Small 8 (2012) 1130.

[24] M .F.L.D. V older, S.H. Tawfick, R.H. Baughman, A .J . Hart, Carbon nanotubes: present and future commercial applications, Science 339 (2013) 535.

[25] D. R obati, S. Bagheriyan, M. Rajabi, O. M oradi, A .A. Peyghan, Effect of electrostatic interaction on the methylene blue and methyl orange adsorption by the pristine and functionalized carbon nanotubes, Phys. E. 83 (2016) 1-6.

[26] X. Zhang, Z. Li, K . Liu, L. Jiang, Bioinspired multifunctional foam with self-cleaning and oil/water separation, A dv. Funct. M ater. 23 (2013) 2881-2886. 
[27] Q. Zhu, Y . Chu, Z. W ang, N. Chen, L. Lin, F. Liu, Q. Pan, Robust superhydrophobic polyurethane sponge as a highly reusable oil-absorption material, J. M ater. Chem. A 1 (2013) 5386-5393.

[28] S. Kabiri, D.N.H. Tran, T. A Italhi, D. L osic, O utstanding adsorption performance of graphene-carbon nanotube aerogels for continuous oil removal, Carbon 80 (2014) 523-533. [29] Z. M okarian, R. Rasuli, Y . A bedini, Facile synthesis of stable superhydrophobic nanocomposite based on multi-walled carbon nanotubes, A ppl. Surf. Sci. 369 (2016) 567-575. [30] M . K alin, M . Polajnar, The wetting of steel, D LC coatings, ceramics and polymers with oils and water: the importance and correlations of surface energy, surface tension, contact angle and spreading, A ppl. Surf. Sci. 293 (2014) 97-108.

[31] Z. Chu, S. Seeger, Superamphiphobic surfaces, Chem. Soc. Rev. 43 (2014) 2784. [32] A. Nakajima, T. W atanabe, K. Takai, G. Y amauchi, A. Fujishima, K. Hashimoto, Transparent superhydrophobic thin films with self-cleaning properties, Langmuir 16 (2000) 7044-7047. [33] X. Liu, Y . Liang, F. Zhou, W. Liu, Extreme wettability and tunable adhesion: biomimicking beyond nature? Soft M atter 8 (2012) 2070-2086.

[34] L.H. K ong, X.H. Chen, L.G. Y U, Z.S. W U, P.Y . Zhang, Superhydrophobic cuprous oxide nanostructures on phosphor-copper meshes and their oil-water separation and oil spill cleanup, ACS A ppl. M ater. Interfaces 7 (2015).

[35] L. X u, G. Zhi, J. He, Z. Gang, M echanically robust, thermally stable, broadband antireflective, and superhydrophobic thin films on glass substrates, A CS A ppl. M ater. Interfaces 6 (2014) 9029.

[36] A. M ilionis, E. L oth, I.S. Bayer, Recent advances in the mechanical durability of superhydrophobic materials, A dv. Colloid Interf. Sci. 229 (2016) 57-79. 